\title{
Agôn
}

Revue des arts de la scène

$5 \mid 2012$

L'entrée en scène

\section{Entrer, d'un pas, dans le flux de ce monde}

Une lecture d'Umwelt de Maguy Marin

\section{Claudia Palazzolo}

\section{(2) OpenEdition \\ Journals}

Édition électronique

URL : http://journals.openedition.org/agon/2420

DOI : 10.4000/agon.2420

ISSN : 1961-8581

Éditeur

Association Agôn

Référence électronique

Claudia Palazzolo, "Entrer, d'un pas, dans le flux de ce monde », Agôn [En ligne], 5 | 2012, mis en ligne le 25 janvier 2013, consulté le 01 mai 2019. URL : http://journals.openedition.org/agon/2420 ; DOI : 10.4000 /agon. 2420

Ce document a été généré automatiquement le 1 mai 2019.

Association Agôn et les auteurs des articles 


\title{
Entrer, d'un pas, dans le flux de ce monde
}

\author{
Une lecture d'Umwelt de Maguy Marin
}

\section{Claudia Palazzolo}

1 Dans un passage inoubliable de May B. (1981), le groupe d'hommes et de femmes tente de sortir de l'espace de la scène. Ils apparaissent, l'un après l'autre, à cour, encore plus courbés, plus fatigués, plus chargés de poids qu'au début. Ils semblent enfin avoir tout pris, leurs valises, même leurs habits d'avant. Et pourtant, après avoir traversé la scène de cour à jardin, ils réapparaissent inéluctablement, comme s'ils oubliaient sans cesse quelque chose comme si, malgré la douleur, il leur était impossible de quitter cet espace. Dans Les applaudissements ne se mangent pas (2002), les entrées en scène se font par n'importe quel point de l'espace, latéralement ou depuis le fond. Perpétuellement agressives, elles dessinent un espace du risque, d'une alerte insoutenable et perpétuelle. Dans l'obscurité de Salves (2010), les entrées rapides, cachées, porteuses d'énergies souterraines et vitales, retissent un nouvel espace d'inquiétude.

2 Dans l'œuvre de Maguy Marin, de pièce en pièce, l'entrée en scène est une véritable entrée en matière chorégraphique, dosant poids, rythmes, tempo sur lesquels se construit la pièce dans sa totalité. Dans cet article, nous interrogerons ce geste d'entrer en scène à partir d'une lecture d'Umwelt, créée en 2004 par Maguy Marin, construite précisément sur la répétition obsessionnelle, idéalement infinie, de l'entrée en scène de l'interprète dans l'espace de la scène.

3 Dans son article "L'être en scène, ou l'espace d'action en danse ", Isabelle Launay insiste sur le fait que, lorsqu'on parle de danse, la question privilégiée n'est pas celle de la mise en scène mais plutôt celle de l'être en scène : «[...] l'espace est créé par le geste : quand le danseur entre en scène il reconfigure donc l'espace, change la qualité de l'air. La première mise en scène est peut-être là ? Comment entre-t-on en scène ? ${ }^{1}$ Qu'est-ce que Umwelt nous dit par rapport à l'entrée en scène, concrète ou métaphorique, dans la mesure où en danse le geste et l'action d'entrée en scène peuvent difficilement être dissociées de leur portée symbolique? Umwelt serait-il donc un spectacle sur l'entrée en scène? 
4 Nous proposerons une approche partielle et orientée d'une pièce dont la richesse s'ouvre à de multiples niveaux de lecture. À quel objet se confronter dès lors que le contexte de représentation, les différentes distributions, les moindres variations, rendent difficile toute objectivation? Comme pour toute pièce chorégraphique, l'étude d'Umwelt nécessite des précautions qui concernent la nature ambiguë de l'œuvre en danse, à la fois corps, partition, contexte, d'autant que, pièce majeure résonnant dans le paysage artistique français, Umwelt a suscité, au moment de sa création, un déferlement de réactions et de discours, dont l'abondance est, dans le champ de la danse contemporaine, quasi sans précédent. Prenant comme point de départ le « désœuvrement $~^{2}$ de l'art chorégraphique comme condition essentielle de sa singularité, nous l'envisagerons à la fois comme une " œuvre ouverte », telle qu'Umberto Eco l'a théorisée en littérature, mais qui sera dans ce cas abordée à partir de l'angle spécifique de l'entrée en scène.

Nous essaierons donc de montrer combien, dans Umwelt, le geste d'entrer en scène, ossifié dans sa réduction et amplifié dans la répétition, peut dialoguer avec la figure de l'entrée chorégraphique dont l'œuvre, qui pourrait sembler faire le deuil de la danse, nous paraît au contraire remémorer les structures ${ }^{3}$. Il s'agira alors aussi de faire dialoguer ces gestes, voire ces figures de la danse, pourtant parfois assez lointaines de l'univers de la chorégraphe.

\section{De quelques entrées, au lointain}

6 Avant d'entrer dans le vocabulaire de la tradition théatrale du ballet occidental, le mot «entrée » est associé au domaine du politique et désigne le cortège triomphal qui accompagne l'arrivée d'un souverain dans une ville, l'hommage ou la soumission de cette dernière à son égard ${ }^{4}$. Ce n'est donc pas par hasard que les entrées de la danse, dans le Ballet comique de la Reine, se situant du côté du roi, incarnent l'harmonie et la paix assurée par l'État contre les dangers des « Ténèbres »" Dans le ballet de cour en effet, chaque acte est divisé en un certain nombre d'« entrées » ou épisodes marqués par l'arrivée puis la sortie de l'espace scénique des personnages dansants. Dans le cadre du ballet de cour l'entrée est ainsi l'unité de base, la division ordinaire des actes dont le spectacle est composé6. Mais le terme «entrée » peut aussi définir un passage dansé dans le cadre de genres mixtes (comédie-ballet, tragédie lyrique, opéra- ballet). Dans l'opéra-ballet, par exemple, l'entrée devient un spectacle dans le spectacle, un ballet autonome, qui développe une intrigue plus au moins liée au sujet de l'opéra ${ }^{7}$. Plus tard, en 1787 sa dimension fictionnelle sera mise en évidence par Compan définissant l'entrée comme « un ou plusieurs quadrilles de danseurs qui par leurs pas, leurs gestes, leurs attitudes représentent la partie de l'action générale dont ils étaient chargés ${ }^{8}$.

7 Sans vouloir développer ici la question de l'entrée en danse sous l'Ancien Régime, qui dépasse de loin le cadre de pertinence de cet article, ce qui nous semble intéressant est que, au-delà des variations, même assez significatives de la notion d'entrée de ballet, ce terme permet surtout de différencier les danses théatrales des autres formes de danse, l'entrée étant synonyme de chorégraphie pour le théâtre. Si l'entrée peut supposer nombre de formats chorégraphiques pour le théatre, elle suppose aussi une figuration, assumée par le fait même de pénétrer dans l'espace du théâtre et donc dans le dispositif de la représentation. Dans les recueils, l'entrée peut en effet être définie en fonction de son caractère : sérieuse, grave, bouffonne ou grotesque, ou par le nombre et le sexe des danseurs (Entrée seul, Entrée seul pour une femme, Entrée à deux, Entrée pour un homme 
et pour une femme) et le type de personnage (Entrée pour un matelot, Entrée de paysan) ${ }^{9}$. Par raccourci, nous pourrions donc considérer l'entrée comme un passage dansé, doué d'une certaine autonomie, rythmique, kinesthésique, ponctué par l'entrée et la sortie des danseurs de l'espace de la scène et ayant une dimension figurative. En ce sens, Umwelt serait donc une succession d'entrées en danse, accomplies pour elles-mêmes, où les passages dansés se seraient en quelques sorte densifiés.

\section{Entrer}

8 À l'entrée dans la salle, le dispositif d'Umwelt aveugle le spectateur par sa clarté. Le fond de la scène est occupé par trois rangées de panneaux réfléchissants disposés en quinconce, sorte de miroirs larges d'environ un mètre et qui en occupent presque toute la hauteur. La froideur métallique de leur apparence, ainsi que la pureté de leur forme rectangulaire apparaissent perpétuellement mise en danger par le « vent » continuel qui, soufflant sur ces panneaux, en déforme le cadre tout en brouillant les images qui y sont reflétées.

9 Le plateau se compose de trois zones distinctes. À l'avant-scène, un système de poulies reliées à des guitares par un fil, qui semble agir comme un archet sur les cordes, renvoie au temps du spectacle: celui-ci sera terminé dès que la bobine arrêtera de tourner, renvoyant les instruments au silence. La deuxième zone est constituée par l'énorme espace du plateau déserté par les hommes, mais qui, au fur et à mesure que la pièce se déroule et que le temps passe, se remplit des traces de leur passage, déchets produits par toutes sortes d'activités humaines, de la plus simple à la plus complexe, qui créent au milieu de la scène une énorme poubelle, seul effet de leurs gestes, unique résultat de l'agitation terrestre. Le temps redevient ici linéaire, procédant de cause à effet, presque narratif. Plus nombreuses et plus complexes sont les actions exécutées, plus elles produisent de tels effets sur le monde représenté par cet espace scénique. Le fond de la scène, dévolu aux danseurs, constitue la troisième zone. C'est en effet dans l'espace qui entoure immédiatement les panneaux, avec ces coulisses sur scène, ces couloirs à demi cachés qu'ils dessinent, que les danseurs se meuvent. La majeure partie de la scène restant vide, délaissée par les danseurs, l'espace paraît presque bi-dimensionnel, beaucoup plus large que profond, et les verticales s'imposent, d'abord tracées par les contours des panneaux, puis soulignées par les postures des danseurs. Ainsi, la première entrée en scène des danseurs les verra tous ensemble - neuf personnes aux habits ordinaires, banals, colorés sans plus. Dépourvus de caractéristiques remarquables, ils ne sont ni beaux ni laids, ni vieux ni jeunes, ni élégants ni mal habillés, ni gros ni maigres, différents autant qu'il suffit, sans pour autant que le groupe soit hiérarchisé. Chacun a la même chance que l'autre d'être regardé, d'exister tout court. Il s'agit d'un des nombreux chœurs, ensembles collectifs auxquels Maguy Marin nous a habitués, mais cette fois, ils nous ressemblent davantage. Alignés, debout devant les panneaux, très éloignés du public, ils regardent droit devant eux en direction des spectateurs. Il pourrait s'agir de nos images en miroir. C'est seulement à la fin de la pièce, qu'on les reverra tous ensemble dans une attitude identique.

Dans Umwelt, le danseur entre en scène, d'abord de la manière la plus simple, en avançant tranquillement, un pas après l'autre, debout comme dans le plus ordinaire des déplacements, la marche. La répétition incessante de ce mouvement ordinaire, banal, permet d'en saisir les détails. 
11 Chaque pas prévoit un changement d'appuis qui modifie l'équilibre obtenu par l'effort des muscles gravitationnels réagissant à la force de la gravité et permettant d'être debout. Ainsi, le déplacement d'une jambe, puis les appuis des pieds au sol, sont générés à partir d'un ajustement général de la posture qui tient compte des caractéristiques morphologiques, des habitudes, ainsi que des intentions individuelles. En général, le pas ne suscite qu'un moindre déséquilibre, sans suspension, ni torsion qui ne soient fonctionnelles au geste quotidien de marcher. À ce minimalisme kinesthésique correspond une économie énergétique. Pas d'efforts importants, pas d'alternance entre les régimes toniques perceptibles.. Selon Hubert Godard, la manière d'organiser la posture, propre à chaque individu, donne aussi une couleur spécifique à des mouvements qui semblent similaires dans leur forme, mais dont la perception se différencie ${ }^{10}$.

Dans l'ouvrage Histoires de gestes (2012), Marie Bardet souligne le caractère paradoxal de la marche fonctionnant sur l'alternance entre adhérence du poids au sol et désir de s'en libérer : « Marcher c'est en même temps s'élever et s'ancrer ; laisser une marque et partir 11. » Dans Umwelt, cette caractéristique du mouvement du corps dans la marche peut se transférer aux enjeux de l'entrée en scène elle-même. On n'entre que pour laisser une marque aussi futile soit-elle, avant de disparaître.

Tout en étant contrainte dans un espace aussi restreint, la marche reste un des gestes les plus communs. L'exploration de motifs kinesthésiques au caractère ordinaire, de simples pas de marche, en arrière et en avant, loin d'être inédite, a traversé la danse à plusieurs reprises. Dans l'esthétique post-moderne, certains dispositifs chorégraphiques déclinent précisément des actions ordinaires, revenant à des mouvements simples, dans la quête d'une danse quotidienne et des qualités de mouvement du «corps piéton ». Explorer l'énergie de l'intérieur est une manière de mettre à distance la séduisante virtuosité du mouvement dansé, pour creuser le moindre mouvement et libérer la danse de la patine brillante du spectaculaire. La concentration sur un geste simple, anodin, banal, quotidien, a également été, dans la phase post-moderne, un outil pour explorer, décortiquer les postures, dévoiler la construction des représentations identitaires - classes, genres, cultures. Postures, gestes, mais aussi costumes, accessoires, sont les marques qui attribuent à chaque corps une construction culturelle pour celui qui le regarde. Il se trouve que, dans Umwelt, à travers la posture, le costume, les accessoires, chaque apparition peut être associée à une figure identitaire.

\section{Mettre en scène les coulisses, cet espace seuil entre scène et hors-scène}

Dans Umwelt, les danseurs entrent en quelques pas d'abord dans un espace invisible de la scène, situé derrière les panneaux, puis ils apparaissent entre les panneaux, dans un espace frontière, un seuil entre le dedans et le dehors, enfin ils pénètrent dans l'espace visible de la scène, en se montrant de dos, de face ou de profil. Le nombre de pas nécessaires pour entrer et sortir de scène, à chaque fois, n'occupe que dix temps et, sur cinq seulement, les danseurs sont visibles. L'espace kinesthésique qu'ils dessinent est également très réduit: circonscrit au fond de l'espace scénique autour des panneaux réfléchissants, puisqu'ils se contentent parfois de circuler dans l'étroit couloir situé à l'arrière de ces panneaux, leurs postures ou leurs gestes construisent un espace plus compact, rétréci ou légèrement dilaté. En général, leur espace de mouvement se déplace 
autour des axes verticaux définis par les miroirs. À partir d'un motif chorégraphique de base, ces quelques pas de marche dont les enjeux sont d'avancer, se tourner, reculer, l'espace d'action du danseur subit des variations plus au moins importantes. Toujours répétant l'action qui consiste à entrer dans l'espace de la scène tel que le définissent les miroirs, les danseurs peuvent se plier, se baisser, se rencontrer, se donner un baiser, s'allonger, ou soudain se mettre à courir dans les couloirs entre les panneaux. Les pas autour desquels la danse d'Umwelt se structure restent cependant la trame visible de la partition.

15 Selon qu'un, deux, ou plusieurs danseurs entrent en scène, l'espace, en vision éloignée, se construit en alternance d'inter-espaces pleins et vides. Dans la partie cachée de la scène, cet espace-temps où le danseur se change, ou prend des accessoires, la partition se déroule également de manière incessante, les pas suivant le même tempo. Pour le public, l'entrée en scène est le fait des apparitions discontinues, qui forment une alternance de pleins et de vides, tandis que pour le danseur, Umwelt demande un travail incessant. Sa disparition à la vue du public, derrière les miroirs, marque en effet le temps des changements de costumes ou d'accessoires, réglé dans le moindre détail comme une chorégraphie. Autant dire que, pour le danseur, se savoir caché au regard ne rend pas moins indispensable la précision dans chaque geste, la conscience de soi. Le dispositif théâtral fondé sur la visibilité de l'acteur semble lui-même être mis en question par cette pièce où, lorsqu'il n'y a pas de danseurs visibles, on continue à percevoir leur présence derrière les miroirs. Où commence l'entrée en scène pour le danseur? Où commence le théâtre? Dans un spectacle où la présence du danseur sur le plateau est au fond perpétuelle, les coulisses sont mises en scène et les entrées sont fausses, processus et forme de la création s'enchaînent dans la continuité.

\section{Répéter, énumérer}

Comme dans presque toutes les pièces de Maguy Marin, on pourrait repérer dans Umwelt des vestiges d'une structure classique, un incipit et une conclusion, mais ces deux seuls moments où, au début et à la fin, tous les danseurs regardent le public, sont réduits ici à quelques instants en dehors desquels le motif d'entrer en scène se répétera constamment pendant toute la pièce, traversant un nombre infini de déclinaisons. La répétition de ce motif gestuel de base, ainsi que ses variations, résonne en quelque sorte avec le principe des «Accumulations» élaboré et pratiqué par Trisha Brown, surtout dans la période allant de 1971 à $1979^{12}$. Le principe selon lequel ce mode de composition s'organise, consiste dans la répétition d'un geste ou d'une phrase simple de mouvement, auquel est ajouté un autre geste puis un autre encore, tout en maintenant la lisibilité du premier motif. Pour d'autres raisons, et avec d'autres enjeux, les nouvelles formes chorégraphiques qui ont émergé en France à partir des années 1990 et qui ont été qualifiées de manière arbitraire de "non-danse $»^{13}$ exploitent elles aussi la répétition à partir du principe de l'énumération neutre d'éléments où les postures pourraient être ramenées à des allitérations.

17 Selon leur degré de lisibilité, ou même de complexité, Umwelt décline une écriture du mouvement pouvant engendrer des gestes globaux ou des actions spécifiques, selon que l'expression des sujets ou l'impact de leur faire sur le contexte est mis en avant. Toujours dans l'ouvrage Histoires de gestes, les auteurs Marie Glon et Isabelle Launay mentionnent une définition ancienne du geste (Hugh de St Victor, 1130) qui, dans les études en danse 
assume un rôle central associé à l'action : « Le geste est le mouvement et la configuration des membres du corps approprié à toute action ou attitude ${ }^{14}$. "

Comme dans les anciennes descriptions, nous appréhendons les différentes entrées d' Umwelt par rapport au sexe du danseur qui apparaît, ou par rapport au nombre de danseurs qui apparaissent, par rapport aux accessoires qui leur attribuent une fonction, parfois par la monstration d'un geste ou d'une action appartenant à différents registres de vie allant du privé ou de l'intime (se déshabiller, pleurer, se gratter), à la caractérisation du statut social ou de l'identité culturelle (revêtir une blouse, un tablier, une djellaba), au registre de la rencontre (s'engueuler, s'embrasser, se donner une gifle) ou encore à celui du jeu (faire une cabriole, taper dans un ballon, jouer de la guitare). Aux accessoires anonymes, s'ajoutent des marques qui relèvent du pouvoir, de la société de consommation, de la guerre, du luxe ou de la violence. Il ne s'agit toutefois pas d'indices fictionnels, ni thématiques, mais de possibilités combinatoires de la pluralité, ayant la même valeur sémantiques que les autres éléments. Dans Vertige de la liste, Umberto Eco définit la liste comme mode d'organisation d'éléments dans l'écriture artistique :

Une liste, c'est l'énumération de choses essentielles ou futiles, c'est l'arrangement aléatoire ou structuré d'éléments cohérents ou disparates, c'est un classement toujours provisoire, c'est l'organisation incongrue ou harmonieuse d'objets réels, virtuels, imaginaires ou symboliques, c'est l'entassement volontaire ou hasardeux d'ensembles homogènes, c'est l'accumulation de définitions nécessaires ou chimériques, c'est l'aboutissement dialectique du dialogue utopique entre le rêve et la raison. C'est le vertige qui nous prend devant l'agencement impossible du monde, un processus utilisé par les artistes ${ }^{15}$.

Dans Umwelt, cela ramène surtout à cette manière d'organiser le temps de la perception par fragments ou petites sections autonomes, mais dont les affinités peuvent apparaître jusqu'à la fin obscures. Pourtant, si l'accumulation, l'ajout de petites actions ou de poses, est le mode de développement le plus courant du motif, la variation permet parfois de revenir du complexe au simple. La seule approche temporelle diachronique, axée sur la succession des événements, ne prend pas en compte la présence des entrées simultanées de deux ou plusieurs danseurs, dont l'effet de double est multiplié par les miroirs. Dans la plupart des cas, en effet, les entrées se font à plusieurs, duo, trio, quatuor, dans la simultanéité ou bien suivant des typologies de compositions musicales - la fugue qui reprend un élément déjà énoncé en modifiant une composante mineure, ou encore le canon, lorsqu'une répétition du motif débute lorsque la phrase précédente n'est pas encore complétée. La composition rythmique, son intense musicalité, élaborée ici en totale autonomie par rapport à la composition musicale, est en effet un autre thème qui traverse toute la recherche de Maguy Marin. Formée entre autres par le grand pédagogue Fernand Schirren ${ }^{16}$ à l'école Mudra de Maurice Béjart, la chorégraphe a manifesté depuis ses premières pièces - May B (1981) ou Babel Babel (1982) en continuant par RamDam (1995) ou Waterzooï (1993) - une sensibilité accrue pour l'écriture du rythme qui, associée au traitement du poids, fonde sa qualité chorégraphique. La variation dans la répétition est donc déterminée non seulement par des éléments kinesthésiques ou figuraux, mais sur des modèles musicaux, permettant d'échapper au pur diachronique comme au pur synchronique. 


\section{Nous, au milieu}

20

En reprenant cette laconique définition de la danse par Emmanuel Kant, qui nous semble pourtant bien adaptée à Umwelt : « jeu de figures dans le temps et dans l'espace $»^{17}$, nous souhaitons interroger maintenant la portée symbolique de ce jeu de figures. Le choix du motif unique, en effet, semble situer la pièce dans un minimalisme radical, qui décrit, plutôt qu'il ne représente. Du point de vue plastique, ce paysage abstrait, réduit et épuré, fait penser aux grandes structures de la matière même, avec son alternance de pleins et de vides, de l'infiniment grand à l'infiniment petit. Pourtant, l'entrée en scène pourrait apparaître aussi comme un mode dramaturgique, le noyau d'un discours poétique.

De pièce en pièce, Magny Marin a inventé des mondes au pluriel qui essayent toujours de répondre à la même question, simple, mais essentielle: comment être ensemble, comment partager espace, énergie et ressources? Dans cette logique où l'existence individuelle peut difficilement être pensée en dehors des espaces collectifs, les groupes de danseurs ressemblent parfois à des chœurs dont les dispositions dans l'espace désignent des lignes d'énergie dynamiques allant de la rencontre, à la manipulation ou à la domination. Dans Umwelt, cependant, ce groupe, est mis en aplat. Cet ensemble aligné au lointain, garde le principe d'individuation, évitant la hiérarchie du placement sur le plateau et refusant le pathos de la fusion chorale dans un corps unique. L'environnement est fait d'un ensemble hétérogène d'individus, l'humanité, dont les dynamiques ne sont jamais tout à fait transparentes. L'univers vu de loin donne à voir des choses que la proximité empêche de remarquer. Ce paysage essentiel, ainsi construit, mettant à distance les danseurs, refuse d'utiliser le potentiel séducteur et le corps à corps de la danse, et propose un mode d'interaction qui interroge le spectateur sur sa propre perception du réel.

Portons maintenant notre attention sur ceux qui entrent en scène et sur l'éventuelle dimension figurative de leur geste. L'apparat para-textuel nous a déjà fait savoir que, dans le groupe, il existe des danseurs non professionnels. Comme les autres danseurs, lorsqu'ils entrent en scène pour la première fois sans rien faire, ils regardent le public, droit dans les yeux. Après quoi, à intervalles réguliers, ils entreront à nouveau en scène, simplement pour regarder les spectateurs, en restant debout sans rien faire d'autre. Ces pauses constituent des sortes d'interruptions dans le flux d'actions indifférenciées. La dimension figurative, ici exacerbée et radicalisée par la distance et l'aplat, contraste en quelque sorte avec ce qui constitue un rappel de l'instant présent, celui de la rencontre entre danseurs et public, ainsi qu'avec les traces concrètes du passage des danseurs, remplissant petit à petit le centre de la scène comme une poubelle.

Par ailleurs, certaines figures font l'objet de plusieurs entrées en scène, réapparaissent deux ou plusieurs fois. Au fur et à mesure que la pièce progresse et qu'elles reviennent dans un ordre nouveau, elles peuvent paraître moins isolées et, tout en étant dépourvues de toute logique narrative lisible, leurs successions suggèrent une dérive imminente. Des actions ordinaires - manger une pomme ou une cuisse de poulet - changent de couleur lorsque, dans une autre entrée, on voit la même danseuse cracher la pomme, ou marcher à quatre pattes avec un faisan mort dans la bouche. Certaines saynètes jouent sur les contrastes en créant des situations paradoxales comme porter une arme et un enfant, embrasser quelqu'un en étant menotté. 
Maguy Marin énumère ainsi un répertoire des possibles, tout en laissant ouvertes leurs possibles combinaisons. Dans cet impressionnant catalogue d'individus exclusivement identifiés par leur posture, la moindre histoire n'est visible que par fragments dépourvus de tout contexte, aucun regard ne pouvant se poser dans une telle mise à distance. Ce regard sur la vie, porté de loin et selon une vision d'ensemble qui la fait apparaître comme un fourmillement d'énergie agitée, où toute histoire, au fond, est perdue au milieu des autres, amène une dimension presque métaphysique à la pièce. La dimension sérielle, cyclique et inéluctable, ainsi que la réduction de l'espace en fond de scène, pourrait même suggérer une référence indirecte au motif fantasmé de la danse macabre, représentant une chaîne ouverte, avec des personnes de sexe, statut et classe sociale différentes, guidées par la Mort. Mais la dimension universelle de cette variation sur l'entrée en scène ne porte chez Maguy Marin aucun fatalisme.

Le fait d'aborder le collectif, de loin, selon une vision d'ensemble, ne veut pas dire que tout est égal. Dans le flux confus des vies où chaque chose se confond avec une autre et leurs icônes avec les choses elles-mêmes, on est placé face à notre responsabilité de choisir de s'abandonner à l'incessante danse des entrées, ou d'essayer d'aiguiser notre perception et de construire un sens dépassant tant l'histoire individuelle que la parabole. Umwelt semble par ailleurs interroger frontalement l'encadrement, banalisé mais jamais banal, de l'entrée du geste dansé dans l'espace du théâtre, et plus généralement, le geste d'(e s')exposer dans l'espace public, ainsi que les responsabilités et les enjeux qu'il suppose et qui en découlent. Que peut la danse? Que peut-elle faire émerger?, semble se demander Umwelt.

Dans ses pièces suivantes, Turba (2007), Description d'un combat (2009), Nocturnes (2012), Maguy Marin aura su donner un corps théâtral à la pensée conceptuelle, redessiner les frontières de la danse en explorant les ressources propres au champ chorégraphique, et suggérer de percevoir le monde par un regard qui, s'il semble s'éloigner, parvient ainsi à mieux l'appréhender.

\section{BIBLIOGRAPHIE}

ADSHEAD-LANSDAL Jeanne [dir.], Dancing texts. Intertextuality in interpretation, Cecil Court Londol, 1999.

BARDET Marie, « Marcher », in Marie Glon et Isabelle Launay [dir.] Histoires de gestes, Paris, Actes Sud, 2012.

BANES Sally, Terpsichore en baskets. Post-modern dance, Paris, Chiron 2002.

BENOIT Marcel [dir.], Dictionnaire de la musique en France, Fayard 1992.

BLUCHE François [dir.], Dictionnaire du Grand Siècle, Paris, Fayard, 1990.

COMPAN Charles, Dictionnaire de danse, Paris, Cailleau, 1787.

FRANKO Mark, La Danse comme texte. Idéologies du corps baroque, Paris, Kargo, 2005. 
ECO Umberto, Vertige de la liste, Paris, Flammarion, 2009.

FRÉTARD Dominique, Danse et non danse, Paris, Cercle d'art 2004.

GÉRARD Genette, Palimpsestes. La littérature au second degré, Editions du Seuil, 1982,.

GODARD Hubert, « Le geste et sa perception », in Marcelle Michel, Isabelle Ginot, La Danse au XX siècle, Paris, Larousse / Bordas, 2008.

KANT Emmanuel, Critique de la faculté de juger, Paris, Garnier Flammarion, 1995.

LAUNAY Isabelle « Poétique de la citation en danse » in Isabelle Launay, Sylviane Pagès [dir.], Mémoires et histoires en danse, Paris, L'Harmattan, 2010.

LE MOAL Philippe (sous la direction de), Dictionnaire de la danse, Larousse 2007.

POUILLAUDE Frédéric, Le Désœuvrement chorégraphique. Etude sur la notion d'œuvre en danse, Vrin, 2009.

\section{NOTES}

1. Isabelle Launay, "L'être en scène, ou l'espace d'action en danse ", in "Théâtre et danse - Un croisement moderne et contemporain ", Études théâtrales, nº 47 et 48, 2010.

2. Voir à ce propos Frédéric Pouillaude, Le Désœuvrement chorégraphique. Etude sur la notion d'œuvre en danse, Paris, Vrin, 2009.

3. Pour l'application de cette notion définie dans le champ de la danse, voir aussi Isabelle Launay, "Poétique de la citation en danse », Mémoires et histoires en danse, Paris, L'Harmattan, 2010, p. 10 ; et Janet Adshead-Lansdal [dir.], Dancing texts. Intertextuality in interpretation, London, Cecil Court, 1999.

4. Francois Bluche [dir.], Dictionnaire du Grand Siècle, Paris, Fayard, 1990.

5. Voir à ce propos Mark Franko, La Danse comme texte. Idéologies du corps baroque, Kargo, 2005, pp. 143-174.

6. Marcel Benoit [dir.], Dictionnaire de la musique en France, Paris, Fayard ,1992.

7. Raoul-Auger Feuillet, Recueils de danse, contenant un très grand nombre d'entrées de ballets de M. Pecour,

tant pour hommes que pour femmes, dont la plus grande partie ont été dansées à l'Opéra, recueillies par M Feuillet Maître de danse, Paris, 1704.

8. Charles Compan, Dictionnaire de danse, Paris, Cailleau, 1787.

9. Philippe Le Moal [dir.], Dictionnaire de la danse, Paris, Larousse 2008.

10. Hubert Godard, "Le geste et sa perception », La Danse au XX $X^{e}$ siècle, Larousse / Bordas, pp. 224-229.

11. Marie Bardet, « Marcher », Histoires de gestes, Paris, Actes Sud, 2012, p. 56.

12. Sally Banes, Terpsichore en baskets. Post-modern dance, Paris, Chiron 2002.

13. Dominique Frétard, Danse et non danse, Paris, Cercle d'art, 2004.

14. Marie Glon et Isabelle Launay, Histoires de gestes, Paris, Actes Sud, 2012, p. 16.

15. Voir Umberto Eco, Vertige de la liste, Paris, Flammarion, 2009.

16. Fernard Schirren, Lee Rythme primordial et souverain, Bruxelles, Editions Contredanse, , 1996 (réed. 2011).

17. Emmanuel Kant, Critique de la faculté de juger, Paris, Garnier Flammarion, 1995, p. 204 ; p. 313. 


\section{RÉSUMÉS}

Cet article propose une lecture d'une pièce de Maguy Marin, Umwelt sous le prisme du geste d'entrer en scène et des figures de l'entrée en danse. Partant de l'usage ancien du terme « entrée de ballet ", désignant en sens large les passages dansés destinés au théâtre, l'article aborde cette dramaturgie des entrées d'Umwelt en analysant leur matière kinéstésique, le dispositif où elles se situent, leur temporalité mais aussi leur figurabilité, ainsi que le décalage entre action d'entrer et réception des entrées par les spectateurs. Déclinant ainsi l'entrée de la danse dans l'espace du théâtre, la pièce questionne la représentation et se révèle comme une formidable expérience de la perception, où le spectateur se voit surtout poussé d'aiguiser le regard et choisir sa place face à l'accumulation d'images et de traces qui font la pluralité fourmillante de ce monde.

\section{INDEX}

Mots-clés : danse, Marin (Maguy), ballet, réception

\section{AUTEUR}

\section{CLAUDIA PALAZZOLO}

Claudia Palazzolo est maître de conférences en Arts du Spectacle (théâtre-danse), à l'Université Lyon2 Lumière. Ses recherches portent sur l'étude de la représentation dans la danse, l'histoire culturelle de la danse envisagée sous l'angle de la réception critique, et plus récemment, l'intertextualité en danse. L'ouvrage, Mise en scène de la danse aux Expositions de Paris : une fabrique du regard, paraîtra en Mai 2013, chez L'œil d'Or. 